\title{
The Effect of Diffusion on the Hydrotreatment of n-C16: A Novel Method to Calculate the Weisz-Prater Number
}

\author{
Junhao Sun ${ }^{1}$, Chao $\mathrm{Mu}^{1}$, Yi Li ${ }^{1}$, Yujun Zhao ${ }^{1}$, Shengping Wang ${ }^{1}$, and Xinbin $\mathrm{Ma}^{1}$ \\ ${ }^{1}$ Tianjin University
}

November 16, 2020

\begin{abstract}
Weisz-Prater number (NW-P) is often applied to assess the internal diffusion effect in heterogeneous catalytic reactions. However, the traditional calculation method with excessive empirical reference values affects the accuracy remarkably. A series of $\mathrm{Pt} / \mathrm{HPMo} / \mathrm{SBA}-15$ catalysts with the pore size as a single variable were prepared to calculate the NW-P with a developed model combining the diffusion-reaction kinetic method. Utilizing dimensionless variables, internal effectiveness factor $(\eta)$ and Thiele modulus ( $\left.\Phi_{-} n\right)$, and the apparent activities over catalysts with different diffusion capacity, NW-P is obtained with improved accuracy. For the diffusion effect on the hydrotreatment of n-C16, according to the more precise NW-P, the pore size should be not less than $10 \mathrm{~nm}$ to avoid the step-limitation of internal diffusion in the premise of adequate acid sites. Using the novel method, a conclusion is drawn that the formation of $\mathrm{m}-\mathrm{i}-\mathrm{C} 16$ is more susceptible to internal diffusion than the consumption of n-C16.
\end{abstract}

\section{Hosted file}

Manuscript1109.pdf available at https://authorea.com/users/375925/articles/493105-theeffect-of-diffusion-on-the-hydrotreatment-of-n-c16-a-novel-method-to-calculate-theweisz-prater-number 